\title{
Demonstration of theoretical and experimental simulations in fiber optics course
}

Tianfu Yao, Xiaolin Wang, Jianhua Shi, Bing Lei, Wei Liu, et al.

Tianfu Yao, Xiaolin Wang, Jianhua Shi, Bing Lei, Wei Liu, Wei Wang, Haojun $\mathrm{Hu}$, "Demonstration of theoretical and experimental simulations in fiber optics course," Proc. SPIE 10452, 14th Conference on Education and Training in Optics and Photonics: ETOP 2017, 1045204 (16 August 2017); doi: 10.1117/12.2264211

SPIE Event: 14th Conference on Education and Training in Optics and Photonics, ETOP 2017, 2017, Hangzhou, China 


\title{
Demonstration of theoretical and experimental simulations in fiber optics course
}

\author{
Tianfu Yao*, Xiaolin Wang, Jianhua Shi, Bing Lei, Wei Liu, Wei Wang and Haojun Hu \\ College of Opto-electronic Science and Engineering, National University of Defense Technology, \\ Changsha 410073, China
}

\begin{abstract}
"Fiber optics" course plays a supporting effect in the curriculum frame of optics and photonics at both undergraduate and postgraduate levels. Moreover, the course can be treated as compulsory for students specialized in the fiber-related field, such as fiber communication, fiber sensing and fiber light source. The corresponding content in fiber optics requires the knowledge of geometrical and physical optics as background, including basic optical theory and fiber components in practice. Thus, to help the students comprehend the relatively abundant and complex content, it is necessary to investigate novel teaching method assistant the classic lectures. In this paper, we introduce the multidimensional pattern in fiber-optics teaching involving theoretical and laboratory simulations. First, the theoretical simulations is demonstrated based on the self-developed software named "See Fiber Tool" (SFTool) which can be installed in both smart phone with Android operating system and personal computer. SFTool tool covers the fundamental calculations relating to transverse modes, fiber lasers and nonlinearities and so on. By comparing the calculation results with other commercial software like COMSOL, SFTool shows high accuracy with high speed. Then the laboratory simulations are designed including fiber coupling, Erbium doped fiber amplifiers, fiber components and so on. The simulations not only supports students understand basic knowledge in the course, but also provides opportunities to develop creative projects in fiber optics.
\end{abstract}

Keywords: Education in optics and photonics, Fiber optics, Theoretical simulation, Laboratory experiment

\section{INTRODUCTION}

In the past decades, fiber optics has developed significantly thanks to the wide applications in communication ${ }^{1}$, laser generation $^{2}$, sensing ${ }^{3}$ and so on. In the case of fiber communication, the capacity based on single mode fiber (SMF) has reached up to $100 \mathrm{Tbit} / \mathrm{s}$ in laboratory, which is closed to the theoretical limit ${ }^{1}$. In another aspect, fiber lasers with high brightness have become available at power levels that make them prime candidates in cutting, engraving, soldering and many other applications normally addressed by solid-state lasers or semiconductor lasers ${ }^{4}$. And with the ever-increasing advances in high-power laser diodes (LDs), fiber design and fabrication, the future for fiber-based light sources looks bright and very interesting with plenty of opportunities for further development and interesting new concepts. Thus to help students understand the basic and important knowledge on fiber optics has become one of the most significant target in the fiber optics curriculum. Through tens of years' education for undergraduate and postgraduate students, we find the traditional classroom teaching limits the innovative thinking and creativity of students.

In order to encourage student to build the abilities on independent thinking and active learning, we adapt to employ the theoretical and laboratory simulations associated with the classroom teaching. To simulate the fiber optics parameters theoretically, one could normally write the code through commercial software, such as Matlab, COMSOL, RP Photonics and so on. However, all the current software is difficult for students with weak base of theory, due to the requirement on programming or unfriendly interface. In that case, it is necessary to develop a software on fiber optics which can be employed directly like a calculator. Recently, a simulation tool named "See Fiber Tool" (SFTool) has been developed and applied in fiber optics curriculum to simulate the relevant knowledge theoretically at the first time $e^{5}$. Based on SFTool, not only teachers and students, but also researchers and techniques can easily calculate and design the fiber optics parameters, which promotes the development on fiber optics theory and the industrialization on fiber related components. By taking advantages of laboratory condition, we have developed a series of experiments to train operational capacity of students as well as hence the understanding of the physical processes.

*yaotianfumary@163.com; phone 0086 0731-84574749

14th Conference on Education and Training in Optics and Photonics: ETOP 2017, edited by Xu Liu,

Xi-Cheng Zhang, Proc. of SPIE Vol. 10452, 1045204 - @ 2017 ICO, IEEE, OSA, SPIE

CCC code: $0277-786 \mathrm{X} / 17 / \$ 18 \cdot$ doi: $10.1117 / 12.2264211$ 
The laboratory simulations include the power coupling from free-space LD to SMF, Erbium doped fiber amplifier (EDFA), wavelength division multiplexing (WDM), and fiber passive components. By combining the theoretical simulations and practical operation together with the classroom teaching, we have helped student to understand significant contents in fiber optics, while improving the ability on analyzing and solving relevant technical problems independently.

\section{FUNCTIONS IN SFTOOL}

Based on the physical equations in the fiber optics curriculum, SFTool with friendly interface allows for direct theoretical simulation by typing in the required parameters. Considering the convenience, SFTool has two versions with the same functions which are for personal computer (PC) and smart phone (SP) with windows and Android operating systems, shown in Fig.1 (a) and (b) separately. In the PC version, one could find the supported base and sub-class functions if there is on the left side and top respectively, while the input parameters and output results are displayed as the main part of the interface. In the SP version, the first interface shows only the base functions, while the input parameters and output results are designed in the secondary interface. In both versions, users can save the calculation results including data and figures in both versions. To help users understand the calculation better, the applied physical equation are listed beside the result. Until now, the functions in SFTool covers laws of optical refraction and reflection, laser characteristics, fiber nonlinearities, fiber dopant concentration, fiber attenuation, transverse modes and profilers in fiber, beam quality and fiber bending loss. In the following sections, we will introduce the main functions in SFTool based on the PC version.

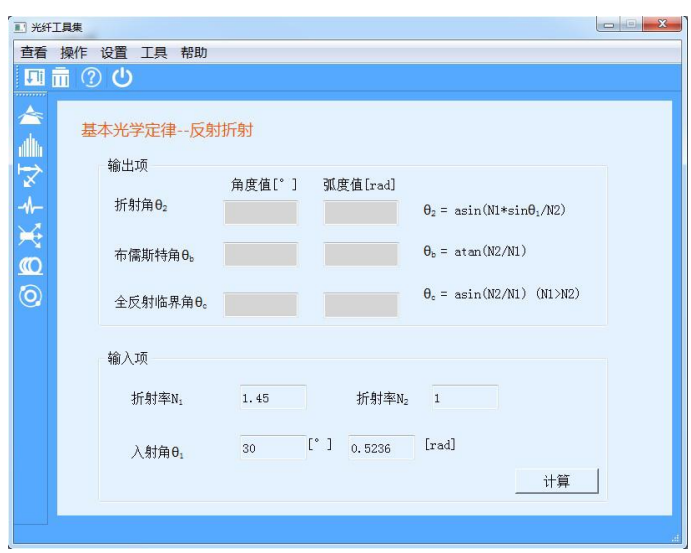

(a)

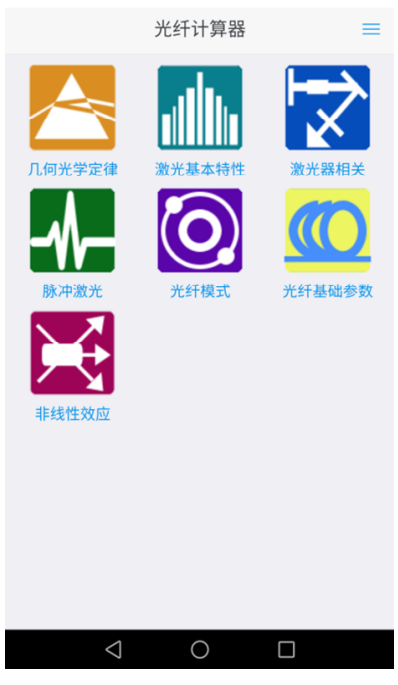

(b)

Fig. 1 Interfaces of SFTool installed in (a) PC and (b) SP.

\subsection{Refraction and reflection laws}

The refraction and reflection laws are the basic knowledge in fiber optics. Due to the total reflection, the optical fiber the optical beam is guided in the fiber core with higher refractive index than that of cladding. The numerical aperture (NA) determines the maximum acceptance angle of a fiber, which can be derived based on the refraction and total reflection laws. Fig. 1 shows the interface of the calculation applied by the refraction and reflection laws. For the fiber core with 1.45 refractive index, the refracted angle in the core is calculated to be 46.469 degree and 0.811 radian, while the critical angle of the total reflection is 34.592 degree and 0.761 radian. 


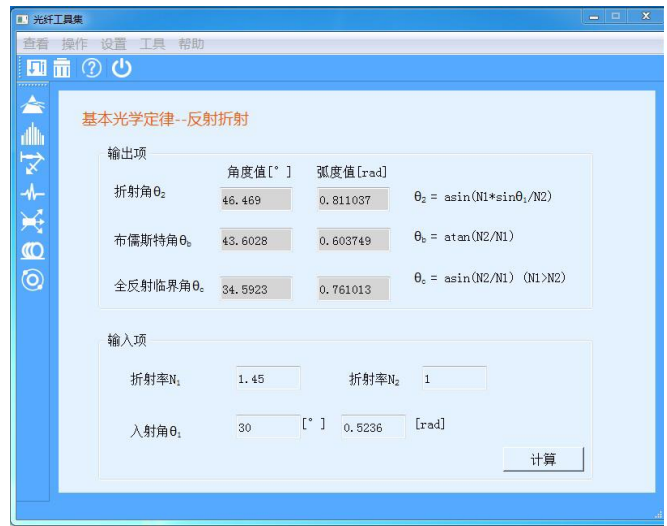

Fig. 2 Simulations on refracted angle and critical angle of the total reflection.

\subsection{Laser characteristics}

The study on laser characteristics would be beneficial not only for understanding laser beam launching and propagating in the fiber, but also for laser generation and amplification in fiber lasers and amplifiers. Firstly, SFTool enables the calculation on the laser frequency, coherent time and length based on the given laser wavelength, frequency spectrum width and wavelength spacing. As a coherent light, laser with excellent monochromaticity benefits numerous applications in dispersion and diffraction fields, thanks to the long coherent time and length. Fig.3 shows calculated results with the set parameters.

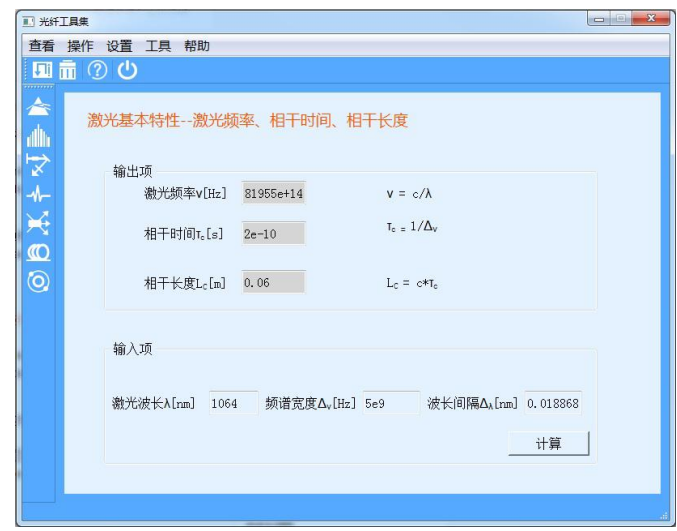

Fig. 3 Simulations on laser frequency, coherent time and length.

Secondly, one could calculate the longitudinal mode spacing and optical conversion efficiency, quantum defect and small signal gain of laser cavity. The longitudinal mode spacing is determined by the refractive index of the laser medium and cavity length. For the laser applied in the optical communication, the material dispersion restrict the bandwidth due to the resulted pulse distortion. To minimize the material dispersion, the narrow linewidth laser with single or few longitudinal mode is preferred. In addition, the temperature would affect the output spectrum of the laser cavity due to the change of the refractive index of the laser medium. Students could understand the design principle of the narrow linewidth laser through the simulation, as well as the matters needing attention for practical laser operation like cooling and so on. The interface of the longitudinal mode spacing calculation is shown in Fig.4, where the input parameters are defined to be 1.45 of refractive index and $10 \mathrm{~m}$ of the cavity length. 


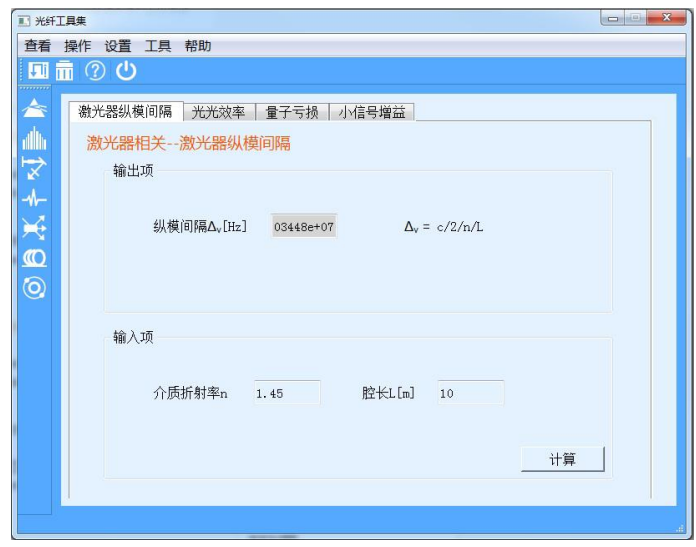

Fig. 4 Simulation on longitudinal mode spacing.

For high power laser applications, the optical conversion efficiency and quantum defect relate to heat generation, while the thermal effects hindering further power scaling. One of the advantages of fiber laser is higher optical conversion efficiency than other kinds of laser. Besides, quantum-defect depression could be another way to reduce the generated heat by cutting down the wavelength difference between pump and signal or increasing the signal wavelength. Fig 5. (a) and (b) shows the simulations on the conversion efficiency and quantum defect separately. In addition, the characteristics of pulse laser has been included in the function. Users could calculate pulse energy and peak power by setting the input pulse width, repeat frequency and average power.

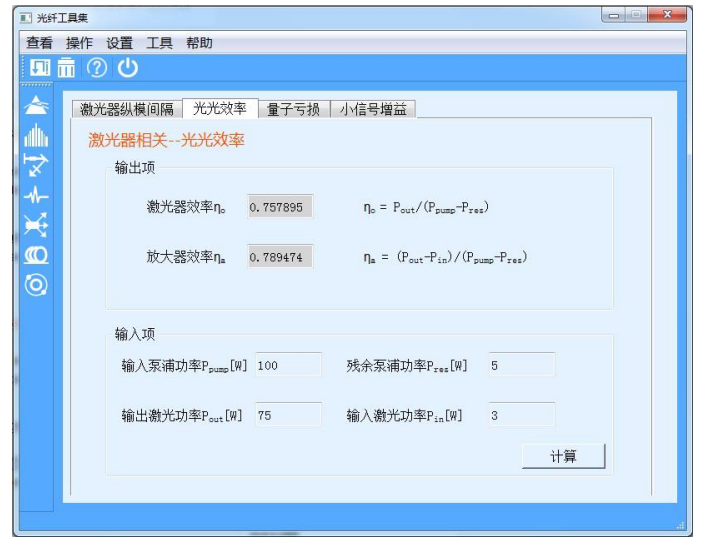

(a)

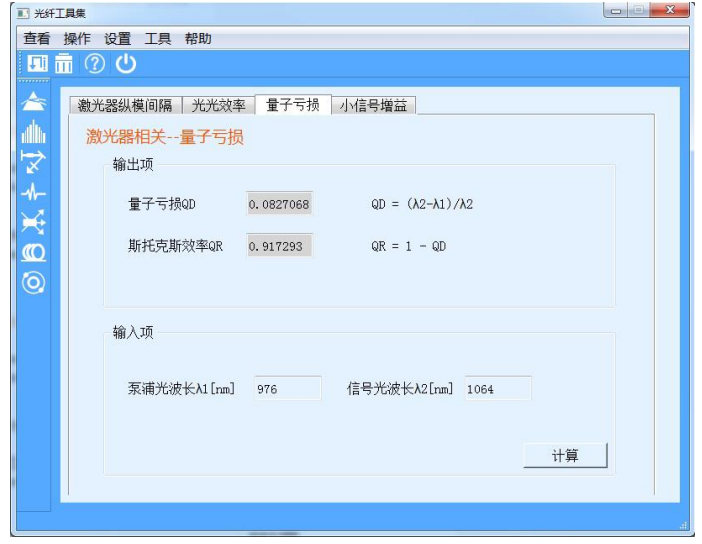

(b)

Fig. 5 Simulations on (a) optical conversion efficiency and (b) quantum defect.

\subsection{Fiber nonlinearities}

Thanks to the thin and long structure, optical fiber with softness, compactness has been attractive in numerous applications. Moreover, the special structure also brings about the appearance of nonlinearities which is desired for novel wavelength generation in one aspect and unwanted for power scaling in another aspect. For both applications, analysis on the fiber nonlinearities would be necessary. SFTool helps users to simulate the fundamental parameters related to nonlinearities, including stimulated Brillouin scattering (SBS), stimulated Raman scattering (SRS), nonlinear phase shift and four wave mixing.

As shown in Fig. 6 (a), the SBS gain coefficient, threshold and wavelength in the fiber amplifier is calculated. The process of SBS can be described classically as the inelastic scattering, where the pump light generates an acoustic wave through electrostriction. The acoustic wave in turn modulates the refractive index of the medium. This pump-induced index grating scatters the pump light through Bragg diffraction. Scattered light is downshift due to the Doppler shift related with a grating moving at the acoustic velocity. The inelastic scattering can be viewed quantum mechanically as if annihilation of a pump photon creates a downshift photon and an acoustic phonon simultaneously. According to the definition of the peak SBS gain coefficient, the input parameters are electrostrictive constant, refractive index, density 
and acoustic velocity of the material, phonon lifetime and pump wavelength. The threshold of SBS in the fiber amplifier depends on the effective mode area of the fiber, polarization factor, fiber length, and the gain of the amplifier. The SBS wavelength corresponding to the peak SBS gain coefficient can be calculated with the pump wavelength and SBS frequency shift.

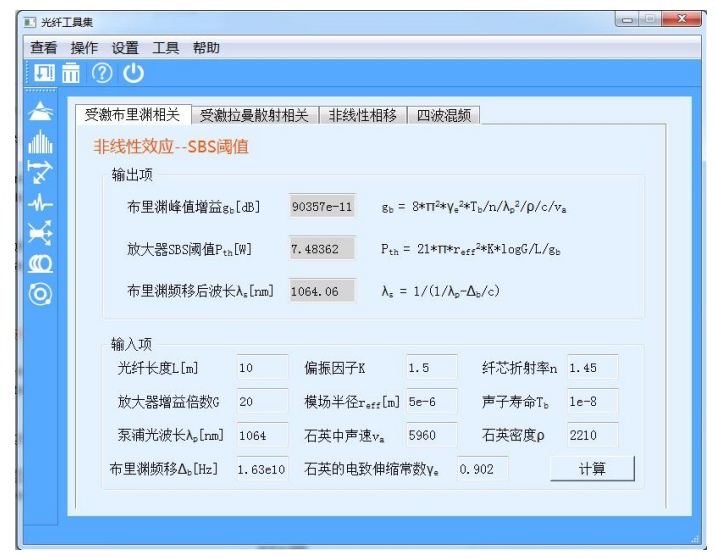

(a)

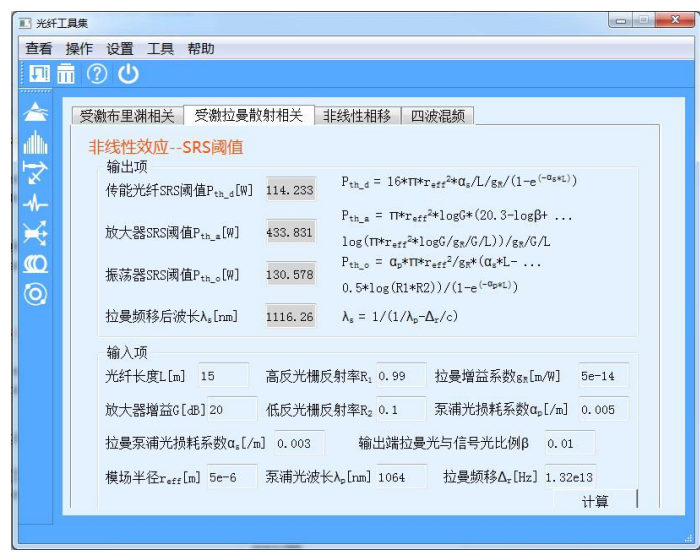

(b)

Fig. 6 Simulations on thresholds of (a) SBS and (b) SRS.

The nonlinearity of SRS is similar to SBS inasmuch as it manifests through generation of a signal wave whose frequency is downshift from that of the pump light by the amount set by the nonlinear medium. However, the process of SRS is another inelastic scattering, where the optical phonons are involved rather than the acoustical phonons. Besides, the frequency shift is larger by three orders of magnitude for SRS compared with that of the SBS. Fig. 6. (b) shows the simulations on SRS threshold in the passive fiber without resonant, the active dopant fiber amplifier and laser cavity. Furthermore, the Raman shift wavelength can also be calculated in that function. The required input parameters are fiber length, Raman gain coefficient, effective mode area, pump wavelength, attenuation factor, Raman frequency shift and other parameters relating to the fiber amplifier and laser cavity, such as amplifier gain and reflectivity. The calculation processes on nonlinear phase shift and four wave mixing are similar to those of SBS and SRS in SFTool, which would not introduced in this paper.

\subsection{Transverse mode}

An optical fiber is a dielectric waveguide that operates at optical frequencies. The propagation of light along a waveguide can be described in terms of a set of guided electromagnetic waves called the transverse modes. For normal step index fiber (SIF) which is weakly waveguide with core radius of $a$, the eigen mode can be regarded as the linearly polarized mode $\mathrm{LP}_{\mathrm{nm}}$ with below expression under polar coordinate.

$$
E_{n m}(r, \phi)=R_{n m}(r) \Phi_{n}(\phi)
$$

where, $\Phi_{n}(\phi)$ determines the degenerate state, while $\cos (n \phi)$ and $\sin (n \phi)$ indicating the even and odd degenerate states respectively.

$$
R_{n m}(r)= \begin{cases}k_{1} J_{n}\left(U_{m} \frac{r}{a}\right) & (0 \leq r<a) \\ k_{2} K_{n}\left(W_{m} \frac{r}{a}\right) & (r \geq a)\end{cases}
$$

where, $J_{n}$ and $K_{n}$ are $n t h$-order first kind and second kind modified Bessel functions separately. $U_{m}$ and $W_{m}$ are defined as

$$
\mathrm{U}_{m}=\frac{2 \pi a}{\lambda} \sqrt{n_{c o}^{2}-n_{m-e f f}^{2}}
$$




$$
\mathrm{W}_{m}=\frac{2 \pi a}{\lambda} \sqrt{n_{m-e f f}^{2}-n_{c l}^{2}}
$$

where, $\lambda$ is the wavelength, $n_{c o}$ and $n_{c l}$ are the refractive index of the core and cladding respectively, $n_{m-e f f}$ is the effective index of the $\mathrm{LP}_{\mathrm{nm}}$ mode. The normalized frequency $\mathrm{V}$ depends on the number of eigen mode, which is given as

$$
V=\frac{2 \pi a}{\lambda} \sqrt{n_{c o}^{2}-n_{c l}^{2}}
$$

By solving the equations above with given fiber parameters, the supported mode profiles with the propagation constant and effective index can be obtained by SFTool as shown in Fig. 7 (a) and (c). The fiber core radius is $15 \mu \mathrm{m}$, the NA is 0.044 with 1.450667 and 1.45 of refractive indices of core and cladding. The laser wavelength is $1064 \mathrm{~nm}$. The calculation takes much less time than those by Matlab and COMSOL. Besides, the mode profiles can be saved as picture in PC or SP.

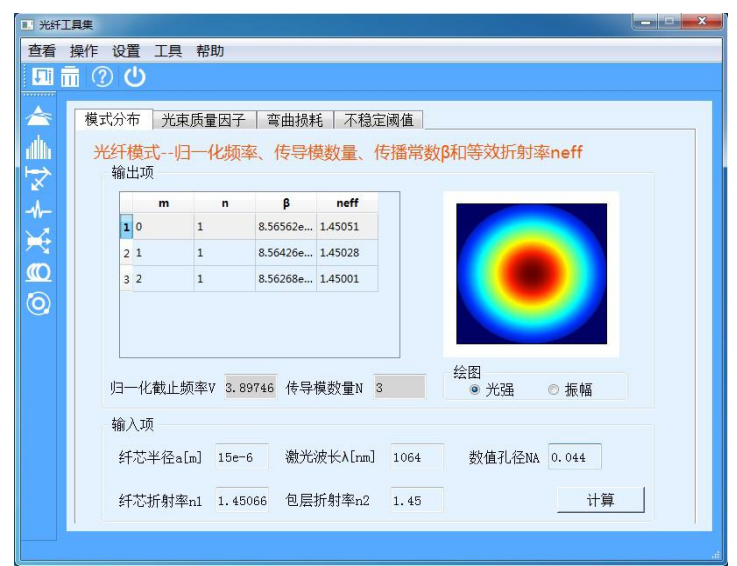

(a)

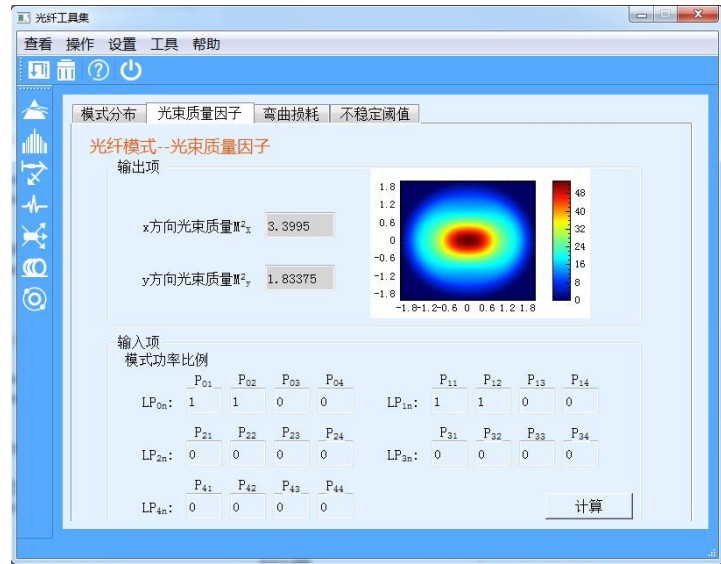

(b)
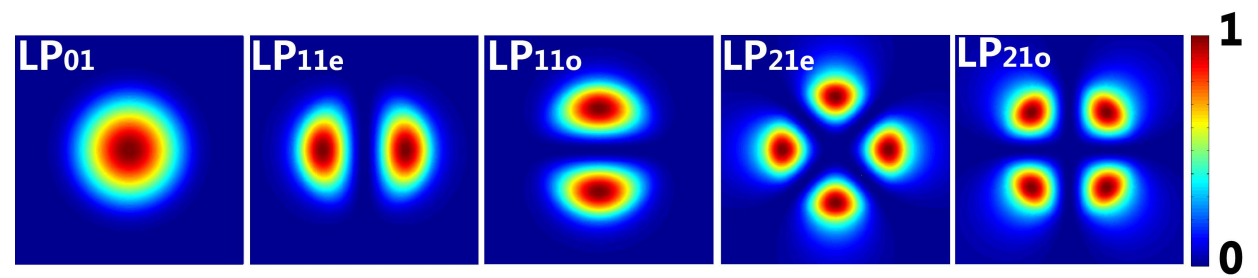

(c)

Fig. 7 Simulations on (a) the propagation constant and effective index of transvers mode, (b) beam quality, (c) modal profiles.

The concepts of brightness and beam quality are generally well known. Indeed the term "high brightness" indicates a high power output and/or a high beam quality. The beam quality of a laser, which is independent of the optical power, corresponds to the degree to which the beam can be focused for a given beam divergence (or convergence) angle. In general, the beam quality can be described by the beam propagation factor (or parameter) $\mathrm{M}^{2}$. For SIF, the beam profile and the quantity of $\mathrm{M}^{2}$ can be observed once the content of eigen modes have be given ${ }^{6}$. As shown in Fig. 7 (b), SFTool enables users to calculate the beam profile and values of $\mathrm{M}^{2}$ at both $\mathrm{x}$ and $\mathrm{y}$ directions.

In practice, due to the limited space, the optical fiber with long length in the equipment are required to be coiled. For multimode fiber, fiber bending leads to the reduction of the incident angle at the interface between the core and cladding. When the incident angle becomes smaller than the critical angle of the total reflection, the mode will propagate from the core to the cladding causing the bending loss. The bending loss can also be regarded as the change of the propagation constants of each mode in multimode fiber. The calculation of the bending losses for the LP01, LP11 and LP21 modes in 
Fig. 7 (a) has been shown in Fig. (8). Under $0.05 \mathrm{~m}$ coiling radius, the bending losses are about 372, 8547 and 4939 $\mathrm{dB} / \mathrm{m}$ for $\mathrm{LP}_{01}, \mathrm{LP}_{11}$ and $\mathrm{LP}_{21}$ modes respectively.

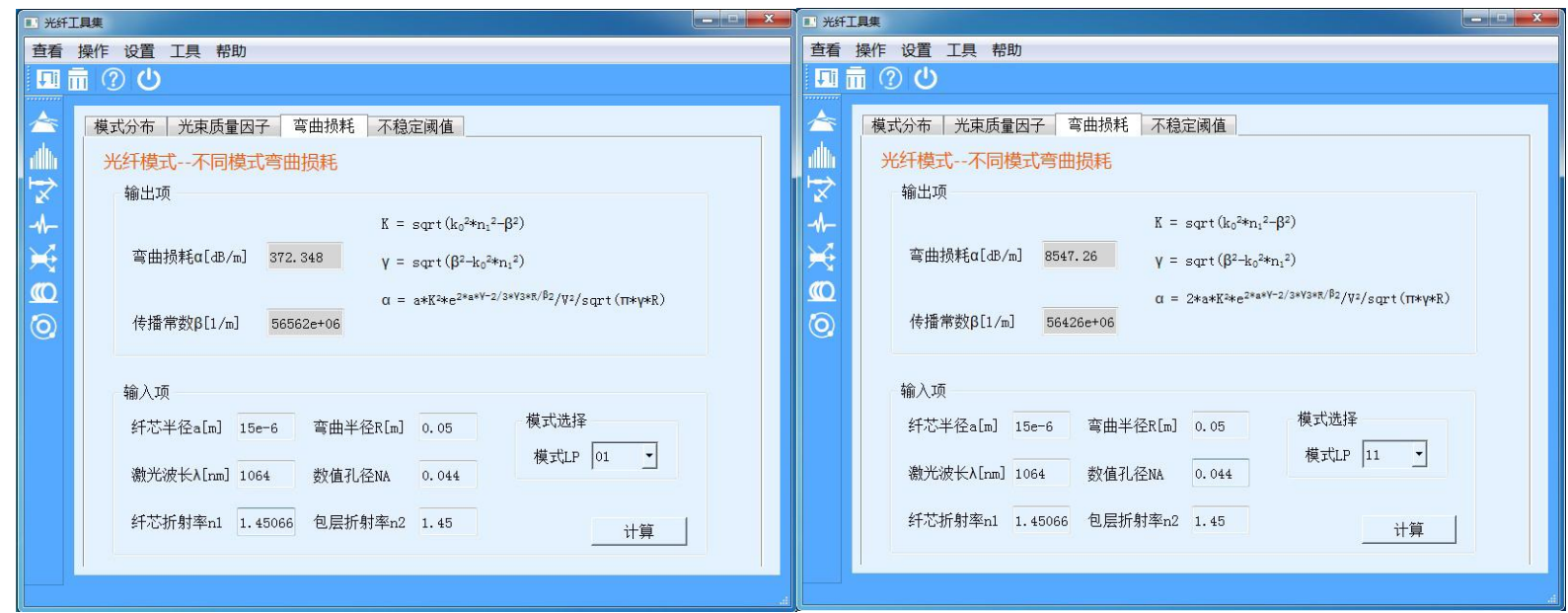

(a)

(b)

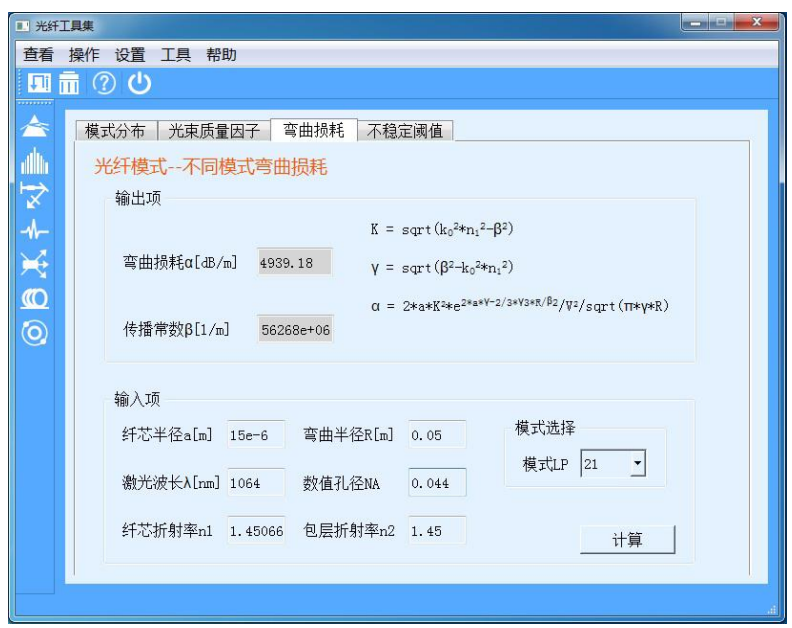

(c)

Fig. 8 Simulations on fiber bending losses for (a) $\mathrm{LP}_{01}$, (b) $\mathrm{LP}_{11}$ and (c) $\mathrm{LP}_{21}$ modes.

\subsection{Active fiber and fiber optics}

Active fiber with rare-earth (RE) dopants including erbium, ytterbium and so on have been investigated and widely applied in optical communication. In additions, active fiber with double-clad structure have also been extensively studied and increasingly developed for power scaling of continuous wave high-power fiber amplifiers and lasers. Typically, double-clad fiber (DCF) consists of a fiber whose so-called inner cladding is surrounded by a lower-index outer cladding and then forms a waveguide around the primary core waveguide. It enables pump-LDs with relatively low brightness to be coupled into the fiber's inner cladding while maintaining a laser output with enhanced brightness. SFTool allows the fundamental calculation on RE dopants concentration and filling factor of a DCF, as shown in Fig. 9 (a) and (b) separately. The RE dopants concentration depends on the small signal absorption coefficient in the core and absorption cross section. However, in experiment, one would measure the small signal absorption coefficient by cladding-pumping scheme firstly. Then the small signal absorption coefficient in the core can be calculated by dividing the claddingpumping small signal absorption coefficient with the filling factor, which is the ratio between the core and cladding areas. Besides, SFTool also provides the calculation on fiber propagation loss, fiber end reflection and amplified spontaneous emission (ASE), which can be operated similarly as above. 


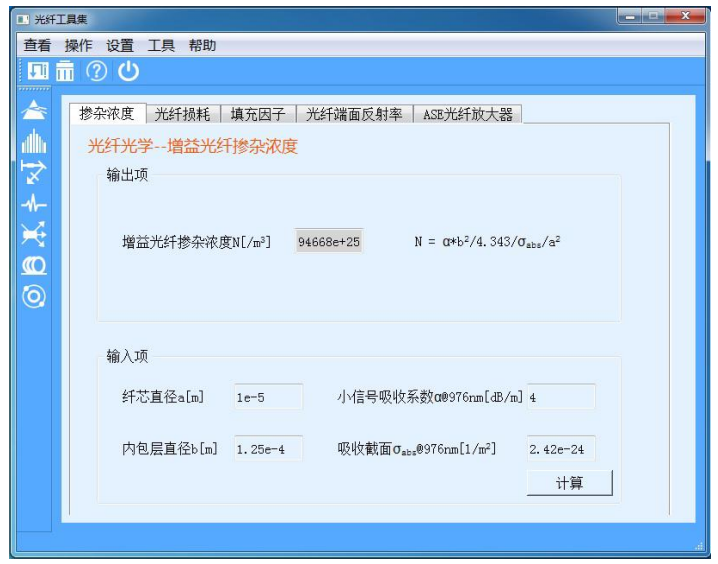

(a)

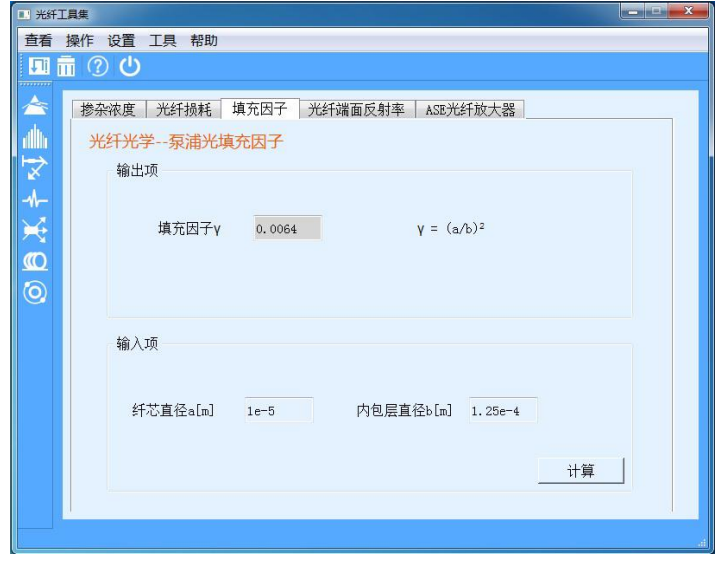

(b)

Fig. 9 Simulations on (a) concentrations and (b) filling factor of active fiber with RE dopants.

\section{LABORATORY SIMULATIONS}

We have developed the fiber-optics experiments consisting of the power coupling from free-space LD to SMF, EDFA, WDM, and fiber passive components. In the power-coupling experiment as shown in Fig. 10 (a) and (b), the students will firstly collimate and focus the laser beam emitted from LD by the optical lenses. Then they will be taught to strip the fiber coating and cleave the fiber end with stripper and cleaver. After fixing the fiber end with clamp near the focal point of the lens, students will learn to adjust the clamp in three dimensions to maximize the output power from the far end of the fiber measured by the power meter. Through the experimental operation, knowledge on the power coupling losses caused by area-mismatch, and NA-mismatch will be enhanced. In this experiment, student can also estimate the NA by measured the output beam diameter on the screen and the distance between the fiber end and the screen. Besides, the reduction on the output power can be observed by bending the fiber with different bending radiuses.

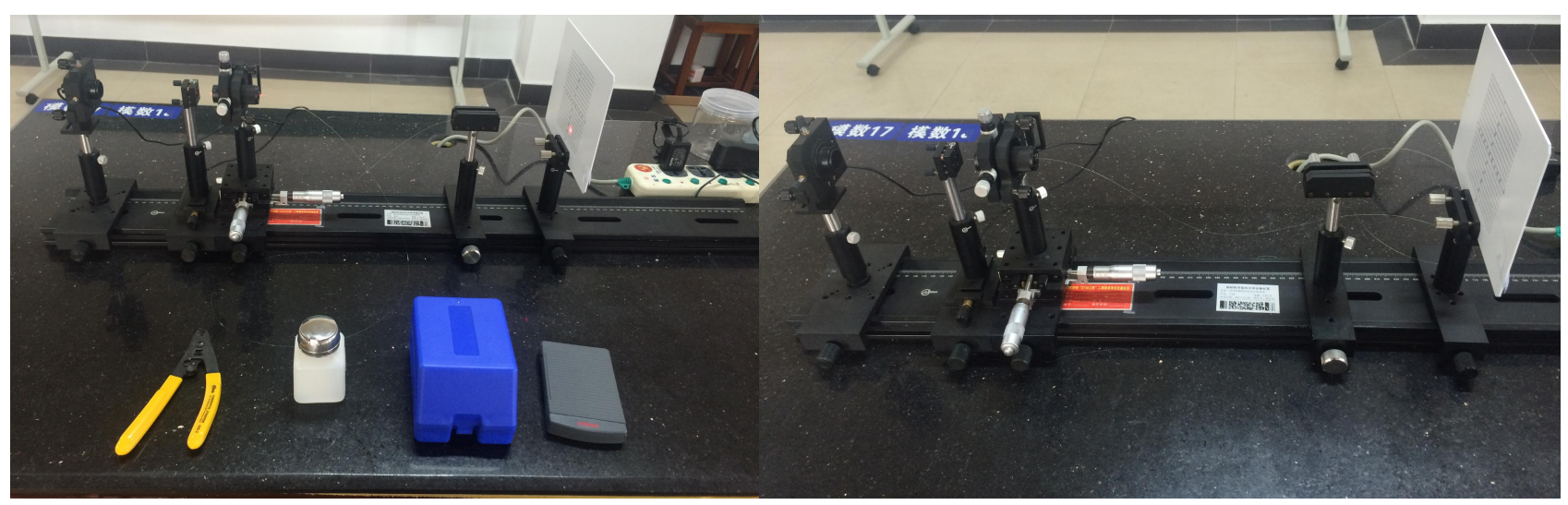

Fig. 10 Experimental setup on power coupling from free-space LD to SMF.

EDFA is applied in the optical communication systems to amplify the attenuated multi-channel optical signals with the same gain. The EDFA is built by Erbium doped fiber (EDF), pump source, WDMs, isolators and filters. The pump wavelength is selected to be 980 and $1480 \mathrm{~nm}$, while the signal wavelength ranging in communication band from 1530 to $1650 \mathrm{~nm}$. By utilizing WDM, the beams at different wavelengths can be combined into one piece of fiber, which is the key to scale the capacity in modern communication system. In EDFA, the pump and signal beams are coupled into EDF through WDM. In addition, the isolators are employed at both input and output ends to suppress the backward beam and protect the pump source. The application of filter at the output end is filtering the generated noise and improving the signal-to-noise ratio. As shown in Fig. 11 (a) and (b), students can compare the output power and spectra from an EDFA by adjusting the input signal power. Besides, student can measure the spectrum before and after a WDM. Also the insertion loss of WDM can be calculated. In addition, the insertion loss and isolation of the isolator can be calculated. 


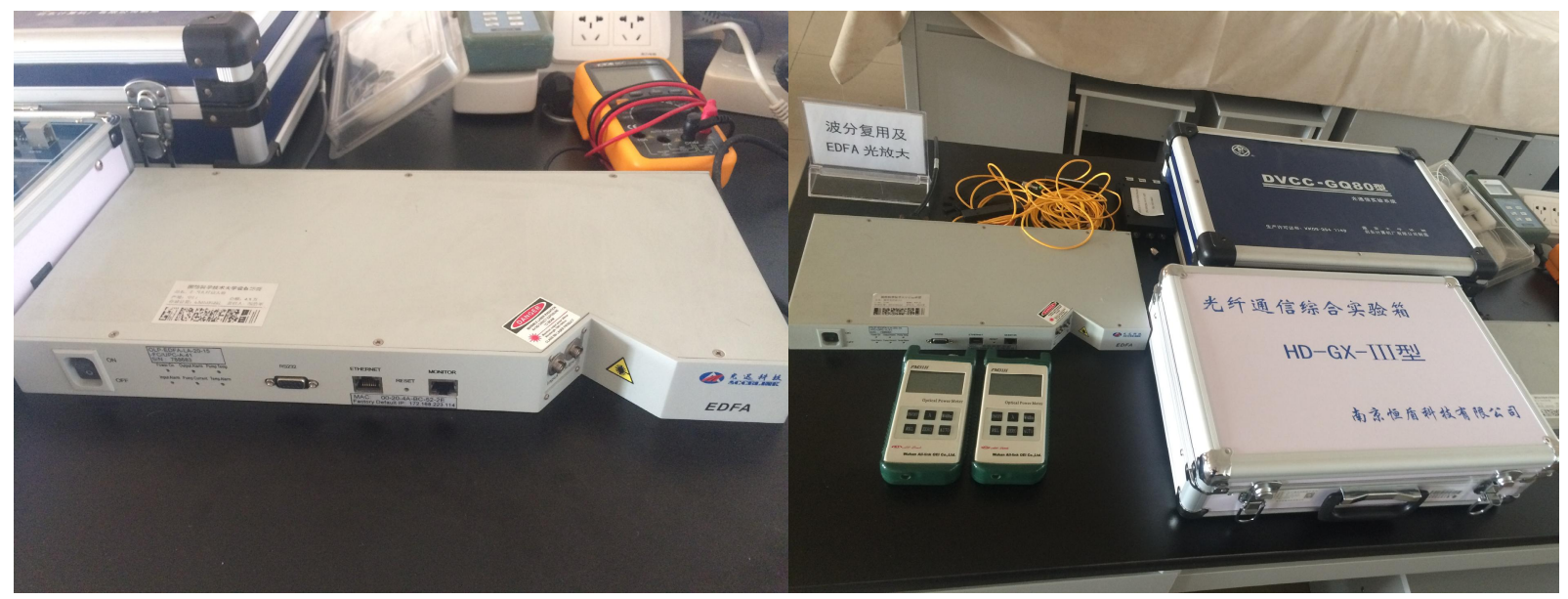

Fig. 11 Experimental setup on EDFA, WDM and fiber passive components.

\section{CONCLUSION}

In this paper, we introduce the theoretical simulator named SFTool for fiber optics education for the first time. The development on SFTool with friendly interface allows users to calculate most of the fiber related parameters conveniently through both PC and SP. The main functions of SFTool include the laws of optical refraction and reflection, laser characteristics, fiber nonlinearities, transverse modes and beam quality, fiber bending loss, active fiber dopant concentration and filling factor, fiber propagation loss, fiber end reflection and ASE. Furthermore, we also design the laboratory experiments about power coupling, EDFA, WDM and fiber passive components. Through the theoretical and laboratory simulations, the knowledge taught in classic classroom teaching has been further enhanced. Moreover, the students can be inspired to think and solve optical problems creatively and independently.

\section{Acknowledgements}

This work is supported by the education and teaching reform research project of College of Optoelectronic Science and Engineering, National University of Defense Technology (Grant No. 2015XY010).

\section{REFERENCES}

[1] Zheng X., Hua N., "Review and outlook of 30 years of optical network development," Tele. Commun. Sci., 32(5), 24-33 (2016).

[2] Bao. X, Chen L., "Recent progress in distributed fiber optic sensors," Sensors, 12(7), 8601 (2012).

[3] Jauregui C., Limpert J., and Tünnermann A., "High-power fibre lasers," Nature Photonics, 7, 861-867 (2013).

[4] Richardson D. J., Nilsson J., Clarkson W. A., "High power fiber lasers: current status and future perspectives," J. Opt. Soc. Am. B, 27(11), B63-B92 (2010).

[5] Wang X., Lv P., Zhang H., Tan S., Xiao H., Duan L., Song Y., Tao R., Shi C., Zhou Y., Ma P., Su R., Zhou P., Xu X., Xu F., "Fiber laser simulation software See Fiber Laser and fiber laser tool SFTool", Chinese Journal of Lasers, 44(5), 0506002 (2017).

[6] Huang L., Guo S., Leng J., Lü H., Zhou P., and Cheng X., "Real-time mode decomposition for few-mode fiber based on numerical method," Optics Express, (23)4, 4620-4629 (2015). 